\title{
1 Can we improve slow learning in cerebellar patients?
}

2 Abbreviated title: Slow learning in cerebellar patients

3 Thomas Hulst ${ }^{1,2,3^{*}}$, Ariels Mamlins ${ }^{1}$, Maarten Frens ${ }^{3}$, Dae-In Chang ${ }^{1}$, Sophia L. Göricke ${ }^{4}$,

4 Dagmar Timmann ${ }^{1 \#}$, Opher Donchin ${ }^{5 \#}$

$5{ }^{1}$ Department of Neurology, Essen University Hospital, University of Duisburg-Essen, 45122 Essen,

6 Germany

$7 \quad{ }^{2}$ Erasmus University College, 3011 HP Rotterdam, The Netherlands

$8{ }^{3}$ Department of Neuroscience, Erasmus MC, 3000 CA Rotterdam, The Netherlands

$9{ }^{4}$ Department of Diagnostic and Interventional Radiology and Neuroradiology, Essen University Hospital,

10 University of Duisburg-Essen, 45122 Essen, Germany

$11{ }^{5}$ Department of Biomedical Engineering, Zlotowski Center for Neuroscience, Ben-Gurion University of

12 the Negev, Beer-Sheva 8410501, Israel

$13 \quad$ \# Authors contributed equally to this work.

$14{ }^{*}$ Corresponding author at: Erasmus University College, Nieuwemarkt 1A, 3011 HP, Rotterdam, E-mail

15 address: hulst@euc.eur.nl (Thomas Hulst)

16 Conflict of interest statement: The authors declare no competing financial interests.

17 Acknowledgements: The study was funded by a grant of the German Research Foundation

18 (DFG TI 239/16-1) awarded to OD and DT and a scholarship from the Essener

19 Ausbildungsprogramm "Labor und Wissenschaft" für den Aerztlichen Nachwuchs (ELAN)

20 supported by the Else Kröner-Fresenius-Stiftung awarded to AM. We would like to thank

21 Beate Brol for her support in the analysis of this experiment. 


\section{Abstract}

23 We tested whether training paradigms targeting slow learning alleviate motor learning deficits

24 of cerebellar patients compared to matched controls using four visuomotor tasks: standard,

25 gradual, overlearning and long intertrial interval. We measured slow learning using

26 spontaneous recovery, the return to learned adaptation after brief wash out of fast adaptation.

27 Spontaneous recovery increased in both groups only in overlearning. A model of adaptation

28 suggested that in controls the increase reflects changes in slow system dynamics. In contrast,

29 because cerebellar participants plateaued more slowly than controls, added trials in models of

30 cerebellar participants increased the level of slow learning. Whereas, under the model, slow

31 learning became more resilient in controls, there was no change in its dynamics for cerebellar

32 participants. Our results and modeling suggest that while residual slow learning does exist in

33 cerebellar patients and can be expressed through increased training trials, the primary

34 cerebellar deficit cannot be improved by overlearning paradigms. 


\section{Introduction}

The cerebellar ataxias are a heterogeneous group of disorders clinically identified by cerebellar dysfunction. Patients exhibit a range of impairments in motor control, including incoordination of eye movements, dysarthria, limb incoordination, and gait disturbances (Mariotti et al., 2005), as well as impairments in the cognitive domain (Schmahmann and Sherman, 1998). While the genetic and pathophysiological underpinnings of many of the cerebellar ataxias are increasingly well understood (Jayadev and Bird, 2013; Matilla-Dueñas et al., 2014), treatment remains a major challenge with genetic therapies being at the horizon for only a subset of genetically defined ataxias (Scoles et al., 2017). Contemporary cerebellar therapy is aimed at alleviating motor symptoms to maintain activities of daily living (ADL), as no curative therapy currently exists (Ilg et al., 2014). Although the consensus is that cerebellar patients benefit from rehabilitation therapy, i.e. physical therapy, speech therapy and occupational therapy, little is known about the mechanisms underlying the improvements and how patients can benefit most (Fonteyn et al., 2014; Ilg et al., 2014). Providing effective care for ataxia patients can be especially challenging, since cerebellar patients suffer from well-known motor learning deficits (Maschke et al., 2004; Tseng et al., 2007) and initial studies suggest a relationship between motor learning deficits and the efficacy of neurorehabilitation programs (Hatakenaka et al., 2012). To develop training programs which are most beneficial for cerebellar patients, one needs to understand which learning abilities remain functional, and whether reduced learning abilities can be improved by certain interventions.

Much research on motor learning has focused on reach adaptation, leading to a wellcharacterized task that serves as good model for more general questions in motor learning and adaptation (Krakauer et al., 2019). In reach adaptation, two learning processes can be distinguished. A fast learning process -- thought to reflect explicit strategic and reinforcement 
60 learning -- and a slow process -- thought to reflect implicit sensory error-based learning

61 (McDougle et al., 2015; Smith et al., 2006). Although the cerebellum has recently been shown to contribute to both processes (Hull, 2020), it is thought to be primarily involved in sensory error-based learning. Indeed, previous work has found that cerebellar patients retain ability to use explicit strategy (Taylor et al., 2010) and reinforcement learning (Therrien et al., 2016). However, there have not been many studies exploring whether slow learning can be utilized by cerebellar patients. Early evidence indicated beneficial effects in gradual introduction of reaching movement perturbations in cerebellar patients (Criscimagna-Hemminger et al., 2010), but the results were not replicated in subsequent work (Gibo et al., 2013; Schlerf et al., 2013). Other paradigms shown to drive slow learning in healthy subjects have not been explored in cerebellar patients. We consider two additional such paradigms. The first is overlearning, continued training after asymptotic performance, which increases retention in healthy subjects as a function of the number of trials trained at asymptote (Joiner and Smith, 2008). The second is to use long intertrial intervals (ITI) between movements. This decreases the rate of learning in healthy subjects, but increases retention (Kim et al., 2015; Sing et al., 2009).

The aim of the present study is to test all the paradigms in the same sets of healthy subjects and cerebellar patients. We tested twenty patients with degenerative ataxia and twenty healthy age- and sex-matched controls on a visuomotor reaching adaptation task under four different training paradigms We tested their effects on the development of slow learning using

80 a behavioral measure called spontaneous recovery. In spontaneous recovery, we measure the 81 tendency of subjects to return to a learned perturbation after application of a short counterperturbation, a phenomenon that is thought to reflect retained slow learning following

83 wash out of the fast component of the initial adaptation (Coltman et al., 2019; Smith et al., 2006). 
85 In addition to the behavioral measure of slow learning, we also characterized it using the two-

86 state model of Smith et al. (2006). Although this model has known limitations (Forano and

87 Franklin, 2020; Petitet et al., 2018; Zarahn et al., 2008), it provides good fit to human

88 behavior and successfully isolates the two main time constants of human learning. By using

89 the model to characterize slow learning, we can gain insight into the changes caused by the

90 behavioral paradigms.

91 Our interest was both in the ability of the different paradigms to drive slow learning in healthy

92 subjects and in the differences between healthy subjects and cerebellar patients. Slow learning

93 is particularly affected in cerebellar patients. We would like to know whether any paradigm

94 that drives slow learning has the ability to leverage the remaining slow learning in hopes of

95 ameliorating the deficit. 


\section{Methods}

\subsection{Participants}

Twenty participants with cerebellar degeneration (9 females, 54.9 years $\pm 10.8(\mathrm{SD})$, range 18

-70 years) and twenty age- and sex-matched participants ( 9 females, 55.2 years $\pm 11.2(\mathrm{SD})$,

100 range $18-71$ years) took part in the study. Cerebellar participants were recruited from the

101 patients attending our ataxia clinic and matched controls were recruited via print

102 advertisements distributed on the hospital campus. Only right-handed individuals were

103 included, as assessed by the Edinburgh Handedness Inventory (Oldfield, 1971). The severity

104 of cerebellar symptoms in the group of cerebellar participants was assessed by one

105 experienced neurologist (DT) and healthy age- and sex-matched controls were examined by

106 AM. Cerebellar symptoms were scored on the International Cooperative Ataxia Rating Scale

107 (ICARS; Trouillas et al., 1997), as well as the Scale for the Assessment and Rating of Ataxia

108 (SARA; Schmitz-Hübsch et al., 2006). The group of cerebellar participants was diagnosed

109 with diseases known to primarily affect the cerebellar cortex (Gomez et al., 1997; Timmann et

110 al., 2009). Three age-matched controls were excluded and replaced due to neurological

111 symptoms on their examination or minor extracerebellar pathology on their MRI. All

112 participants gave informed oral and written consent. The experiment was approved by the

113 ethics committee of the medical faculty of the University of Duisburg-Essen and conducted in

114 accordance with the Declaration of Helsinki. The characteristics of the recruited cerebellar

115 participants and matched controls can be found in Table 1. 


\section{Table 1}

Overview Cerebellar participants and Control participants

\section{Cerebellar participants}

\section{Control participants}

ID Age Sex Diagnosis

$\begin{array}{ccc}\begin{array}{c}\text { Disease } \\ \text { duration }\end{array} & \text { ICARS } & \text { SARA } \\ (\text { total } / 100) & (\text { total } / 40)\end{array}$

ID

Age Sex

\begin{tabular}{|c|c|c|c|c|c|c|c|c|c|}
\hline P01 & 18 & $\mathrm{M}$ & ADCAIII & 18 years & 10.5 & 5 & $\mathrm{C} 01$ & 18 & $\mathrm{M}$ \\
\hline P02 & 47 & $\mathrm{M}$ & ADCAIII & $25+$ years & 40 & 17.5 & $\mathrm{C} 02$ & 43 & M \\
\hline P03 & 50 & $\mathrm{~F}$ & ADCAIII & $25+$ years & 31 & 11.5 & $\mathrm{C} 03$ & 50 & $\mathrm{~F}$ \\
\hline P04 & 51 & $\mathrm{M}$ & SCA14 & $25+$ years & 31 & 11.5 & $\mathrm{C} 04$ & 50 & $\mathrm{M}$ \\
\hline P05 & 51 & $\mathrm{~F}$ & ADCAIII & 19 years & 29 & 12.5 & $\mathrm{C} 05$ & 51 & $\mathrm{~F}$ \\
\hline P06 & 52 & $\mathrm{~F}$ & SCA14 & 22 years & 26.5 & 12 & $\mathrm{C} 06$ & 53 & $\mathrm{~F}$ \\
\hline P07 & 53 & $\mathrm{~F}$ & SCA6 & 2 years & 23 & 9.5 & $\mathrm{C} 07$ & 54 & $\mathrm{~F}$ \\
\hline P08 & 53 & $\mathrm{M}$ & SAOA & 17 years & 40 & 15 & $\mathrm{C} 08$ & 53 & $\mathrm{M}$ \\
\hline P09 & 53 & $\mathrm{M}$ & ADCAIII & 17 years & 36 & 11 & $\mathrm{C} 09$ & 51 & M \\
\hline $\mathrm{P} 10$ & 54 & $\mathrm{~F}$ & SCA6 & 4 years & 30.5 & 10 & $\mathrm{C} 10$ & 56 & $\mathrm{~F}$ \\
\hline $\mathrm{P} 11$ & 56 & $\mathrm{~F}$ & SCA14 & $25+$ years & 28 & 12 & $\mathrm{C} 11$ & 58 & $\mathrm{~F}$ \\
\hline $\mathrm{P} 12$ & 57 & $\mathrm{M}$ & SCA6 & 11 years & 38 & 11 & $\mathrm{C} 12$ & 53 & $\mathrm{M}$ \\
\hline P13 & 57 & $\mathrm{M}$ & SCA6 & 15 years & 28 & 8 & $\mathrm{C} 13$ & 59 & $\mathrm{M}$ \\
\hline P14 & 58 & $\mathrm{M}$ & SAOA & $25+$ years & 63.5 & 22 & $\mathrm{C} 14$ & 61 & M \\
\hline $\mathrm{P} 15$ & 59 & $\mathrm{M}$ & SCA6 & 5 years & 23 & 9.5 & $\mathrm{C} 15$ & 63 & $\mathrm{M}$ \\
\hline $\mathrm{P} 16$ & 60 & $\mathrm{~F}$ & SCA6 & 11 years & 36.5 & 14 & $\mathrm{C} 16$ & 60 & $\mathrm{~F}$ \\
\hline $\mathrm{P} 17$ & 63 & $\mathrm{~F}$ & ADCAIII & 23 years & 33 & 13.5 & $\mathrm{C} 17$ & 66 & $\mathrm{~F}$ \\
\hline P18 & 66 & $\mathrm{M}$ & SAOA & 13 years & 24.5 & 11 & $\mathrm{C} 18$ & 66 & $\mathrm{M}$ \\
\hline P19 & 70 & $\mathrm{~F}$ & SAOA & 7 years & 32.5 & 12.5 & C19 & 71 & $\mathrm{~F}$ \\
\hline $\mathrm{P} 20$ & 70 & $\mathrm{M}$ & SAOA & 16 years & 38 & 15 & $\mathrm{C} 20$ & 67 & $\mathrm{M}$ \\
\hline
\end{tabular}

116 Table 1: Cerebellar participants were age-and sex-matched with the controls on the right side of the table.

117 SCA6 = spinocerebellar ataxia type 6; SCA14 = spinocerebellar ataxia type 14; SAOA = sporadic adult onset

118 ataxia; ADCA III = autosomal dominant ataxia type III; ICARS = International Cooperative Ataxia Rating

119 Scale (Trouillas et al., 1997); SARA = Scale for the Assessment and Rating of Ataxia (Schmitz-Hübsch et al.,

120 2006). Disease duration is years since presentation of the first symptoms.

\section{$121 \quad 2.2$ Task}

122 All participants completed a standard reaching task with visuomotor perturbations. The

123 experimental setup and task have been described previously in other studies from our group

124 (Rabe et al., 2009). In short, participants were seated in front of an upright monitor and could, 
125 with their right hand, move a two-jointed manipulandum freely in the horizontal plane

126 (Figure 1A). Vision of the participant's arm was obstructed by a black cloth. Hand position

127 and velocities were measured in a resolution of $10^{6}$ counts per revolution and a sampling rate

128 of $200 \mathrm{~Hz}$ (DMC-1826; Galil Motion Control). The location of the participant's hand was

129 represented on the monitor by a green dot with a diameter of $5 \mathrm{~mm}$. The origin and target

130 locations were represented by a circle with a diameter of $10 \mathrm{~mm}$, colored red and white

131 respectively. At the start of each trial, the participant's hand was moved towards the origin

132 location by the servomotors connected to the manipulandum. Then, after a delay of 2,000 ms,

133 a target circle appeared at one of three possible target locations, located 10 centimeters away

134 from the origin at an angle of $66^{\circ}, 90^{\circ}$ or $114^{\circ}$ (Figure 1B). Participants were instructed to

135 move the green dot from the origin towards the target with a "quick and accurate movement"

136 as soon as the target appeared. When participants moved the cursor through an invisible

137 boundary located 10 centimeters from the origin, their hand was gently brought to a stop by a

138 simulated cushion, indicating the end of the movement. Following each movement,

139 participants received feedback on whether they hit the target and moved with the correct

140 velocity. The target turned yellow when moving too fast, blue when moving too slow, and

141 green when moving with the correct velocity. Participants moved with the correct velocity

142 when their movement and reaction time fell within a $250 \mathrm{~ms}$ window centered around $500 \mathrm{~ms}$.

143 The $250 \mathrm{~ms}$ window shrunk by $10 \%$ every time a movement had the correct velocity and

144 increased by $10 \%$ when moving too fast or slow, adapting to a participant's individual

145 capabilities. When participants also managed to hit the target, in addition to moving with the

146 correct velocity, a "yahoo" sound was played. 
A

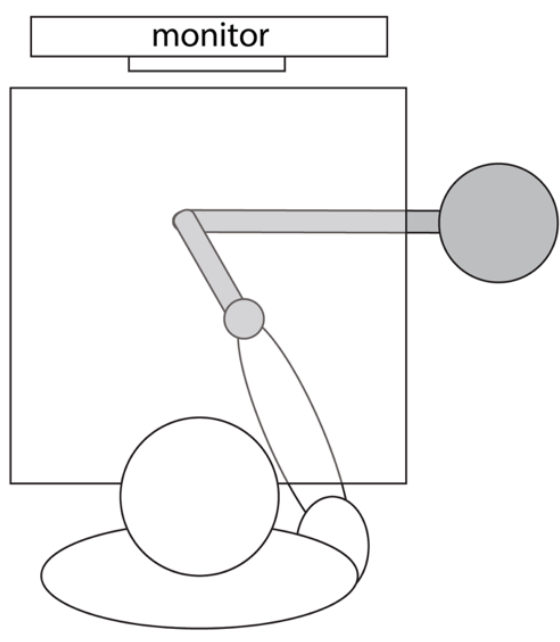

B

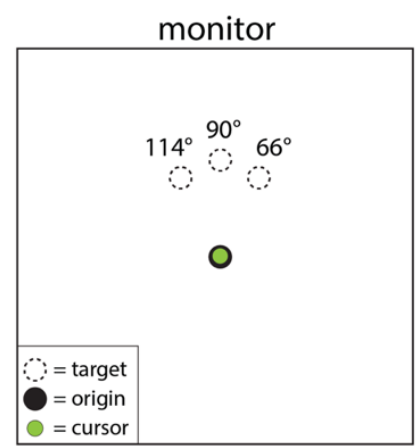

Figure 1: A) Experimental setup. For illustrative purposes the tabletop is pictured here as transparent. In reality the tabletop was opaque to obstruct the view of the hand and robot arm. Additionally, a black cloth was draped over the shoulders of the participant and attached to the table to obstruct vision of the arm. B) Localization of origin and target circles on the monitor. One of the three target circles would pseudo-randomly appear at the start of a movement trial.

The experimental task consisted of 4 different training paradigms. Each paradigm consisted of a baseline set, an adaptation set, and a washout set. All participants completed each of the training paradigms. The order of paradigms was counterbalanced with a Latin-squares design against first-order carryover effects (Williams, 1949). Every paradigm order was completed by 10 participants each (five cerebellar participants and five control participants, Figure 2A). Analysis of the effects of counterbalancing are presented in the supplementary materials. Furthermore, perturbation direction in the adaptation sets was balanced by flipping the direction of the perturbation (clockwise or counterclockwise) in every successive adaptation set. Participants were allowed to take 5- to 10-minute breaks between paradigms, but not after sets within a paradigm. Each baseline set consisted of 135 null trials, in which participants received veridical feedback on hand position, and 15 pseudo-randomly interspersed clamp trials, in which participants received perfect feedback regardless of movement error. Then, depending on the training paradigm, one of four adaptation sets followed. In the standard training paradigm, the adaptation set consisted of 108 adaptation trials, in which a visuomotor 
167 perturbation of $30^{\circ}$ was introduced abruptly, and 12 pseudo-randomly interspersed clamp

168 trials (Figure 2B). The gradual paradigm contained 108 adaptation trials in the adaptation set,

169 where the visuomotor perturbation of $30^{\circ}$ was introduced gradually over the course of the set,

170 increasing linearly each trial. The final 6 trials of the gradual adaptation set were at $30^{\circ}$ of

171 visuomotor perturbation. In addition, 12 clamp trials were pseudo-randomly interspersed

172 (Figure 2C). The overlearning adaptation set consisted of 324 trials with a visuomotor

173 perturbation of $30^{\circ}$ (three times the amount of the standard paradigm) and 36 interspersed

174 clamp trials (Figure 2D). The long intertrial interval (ITI) adaptation set included 120

175 adaptation trials with visuomotor perturbations of $30^{\circ}$, where instead of delay of 2 seconds

176 between each movement, the delay was increased to 15 seconds (Figure 2E). The adaptation

177 set of the long ITI paradigm did not include any clamp trials, thus trial-to-trial forgetting was

178 only dependent on the passage of time. Finally, all adaptation sets were followed by a

179 washout set. The first 12 trials of the washout set consisted of counterperturbation trials,

180 where the direction of the perturbation was flipped from the direction in the adaptation set.

181 Then, 60 clamp trials and 60 null trials followed. 
A

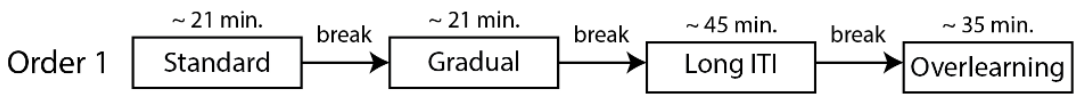

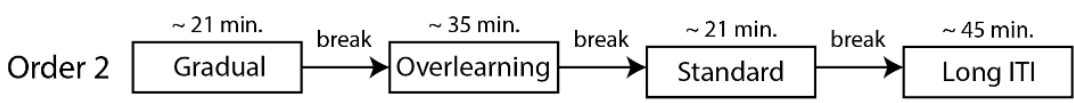

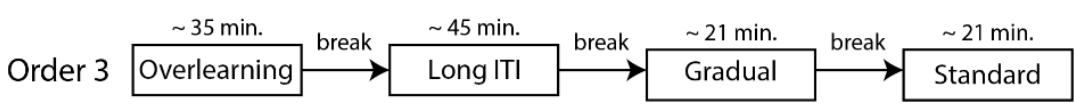

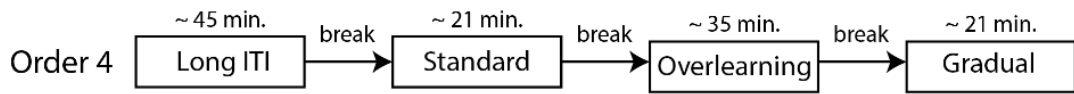

B
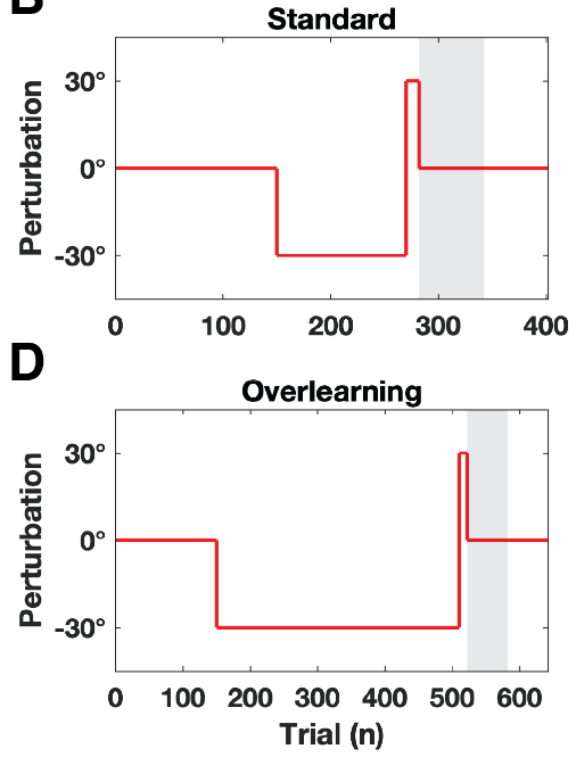

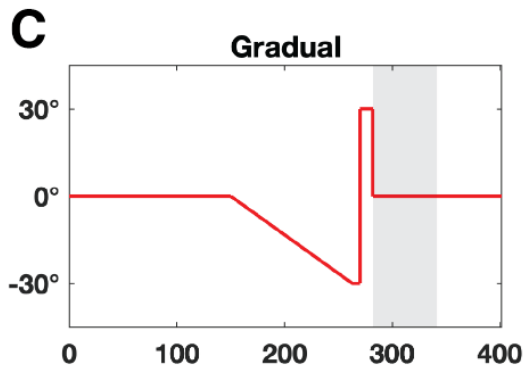

E

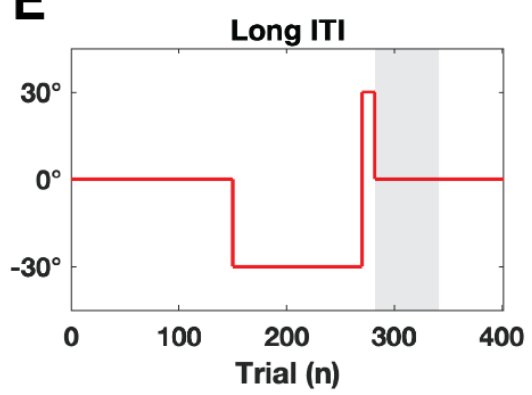

Figure 2: A) Overview of the paradigm orders. B-E) Trial structure of the experimental paradigms. Red line indicates the size and direction of the visuomotor perturbation. Direction of the perturbation is pictured here as clockwise for all paradigms, in reality perturbation direction was counterbalanced within participants. Grey area indicates the block of 60 clamp trials in the washout set. Not pictured are pseudo-randomly interspersed clamp trials during the baseline and adaptation phase.

\subsection{MR imaging}

Cerebellar participants and age-matched controls were examined in a 3T combined MRI-PET

system (Siemens Healthcare, Erlangen, Germany) with a 16-channel head coil (Siemens

191 Healthcare) $[\mathrm{TR}=2,530 \mathrm{~ms}$; $\mathrm{TE}=3.26 \mathrm{~ms}$, $\mathrm{TI}=1,100 \mathrm{~ms}$; flip angle $7 \mathrm{deg}$; voxel size $0.5 \times$

193 voxel-based morphometry analysis was applied to the cerebellum of each participant as

194 described previously (Hulst et al., 2015; Taig et al., 2012). The analysis was automated with

195 an in-house program written for MATLAB 9.4 using the SUIT toolbox (version 3.2) 
196 (Diedrichsen et al., 2009), implemented in SPM12

197 (http://www.fil.ion.ucl.ac.uk/spm/software/spm12).

198

199

200

201

202

203

204

205

206

207

208

209

210

211

212

213

214

215

216

217

218

220

\subsection{Analysis of behavioral data}

Behavioral data was analyzed in MATLAB 9.4 (MathWorks, Natick, USA). Our primary outcome measure was the reaching direction (in degrees) at the end of the movement (i.e., when participants hit the simulated cushion). The reaching direction was calculated by taking the angle between a straight line from the position of movement onset to the target and a straight line from the position of movement onset to hand position at the end of the movement. Movement onset was defined as the first moment when movement velocity exceeded $5 \mathrm{~cm} / \mathrm{s}$. Reaching directions were corrected for movement biases by calculating the average reaching direction in each baseline set and subtracting this from the subsequent adaptation and washout sets of a training paradigm. For ease of interpretation, reaching directions were flipped towards the same direction, regardless of perturbation direction, in all figures and analyses. Furthermore, paradigms were reordered to a canonical order for each participant, starting with the standard learning paradigm, then gradual learning, overlearning and finally the long ITI paradigm, regardless of the order the participant encountered the paradigms. Statistical analyses were conducted using Markov Chain Monte Carlo (MCMC) methods in MATLAB and JAGS 4.3.0 (Plummer, 2003). A mixed-design model (ANOVAlike) was used to estimate the difference in reaching directions between factors. Participant group (cerebellar participant or control participant) was included as a between-subject factor. Movement phase and training paradigm were included as within-subject factors. Movement phases were defined as follows: baseline (all 150 trials in the baseline set), early adaptation (first 30 trials of the adaptation set), late adaptation (final 6 trials of the adaptation set) and recovery (all 60 clamp trials in the washout set). A random intercept for each participant and phase was included as well. The model ran on four separate chains with an adaptation phase 
of 5,000 samples and burn-in phase of 25,000 samples, after which we collected 50,000

samples per chain. The MCMC procedure gives us a posterior distribution of credible parameter values, given the data. The $95 \%$ highest density interval (HDI) contains $95 \%$ of the mass of credible parameter values, where each value within the HDI has a higher probability density than any value outside the HDI. When the HDI falls completely within the Region of Practical Equivalence (ROPE), we accept the null value of the parameter and when the HDI falls completely outside the ROPE, we reject the null value of the parameter. The ROPE was set at $\left[-3.5^{\circ} ; 3.5^{\circ}\right]$ to match the within group variability between subjects. Each parameter using common MCMC diagnostics (Kruschke, 2010). First, trace plots were visually

231 inspected for chain convergence. Next, the effective sample size (ESS), the potential scale reduction factor (PSRF) and the Monte Carlo Standard Error (MCSE) were calculated for all parameters. The PSRF was close to 1 for each parameter (max: 1.0002), MCSE was close to 0

234 for each parameter (max: 0.0001), and median ESS was generally large $(>>5,000)$, indicating 235 convergence of the model run. The model code is available on

236 https://github.com/thomashulst/paper-slowlearning.

\section{$237 \quad 2.5$ State-space modeling}

238 A two-state model of motor learning was fit to the reaching directions of all trials in each 239 individual participant. This classical model was originally presented by Smith and colleagues 240 (Smith et al., 2006) and posits a fast state $\left(x_{t}^{F}\right)$, that learns and forgets quickly, and a slow 241 state $\left(x_{t}^{S}\right)$, that learns and forgets slowly, both adapting to the motor error. The model was fit 242 using a Bayesian approach similar to that in van der Vliet et al. (2018). Details of the model 243 and the Bayesian fitting procedure used to estimate individual subject parameters are

244 available in the supplementary materials. The model code is available on 
246 We examined the internal model parameters to gain insight into the factors driving increased

247 spontaneous recovery in the overlearning paradigm. To this purpose, we compared the

248 estimated learning and forgetting rates of the fast system (BFast and AFast, respectively) and

249 slow system (BSlow and ASlow) across paradigms and groups, both at the level of individual 250 subjects and the population. 


\section{Results}

\subsection{Voxel-based morphometry (VBM)}

253 First, the results of the structural MRI data were analyzed using VBM. Figure 3 displays the

254 difference in gray matter volume per voxel (in t-scores) between healthy participants and

255 cerebellar participants. A resampling procedure (permutation test) was conducted to control

256 the family-wise error rate. The significance threshold was determined to be 3.95 , meaning that

257 voxels with an absolute t-score higher than 3.95 were considered significant. No significant

258 positive differences were found; thus, the figure displays negative t-scores only. The VBM

259 analysis revealed a pattern of cerebellar degeneration in patients largely consistent with prior

260 work (Hulst et al., 2015). The volume loss was largest in the anterior lobe of the cerebellum

261 and the superior part of the posterior lobe (i.e., lobule VI). Cerebellar degeneration of the

262 anterior cerebellum and lobule VI (i.e. the anterior hand area) are associated with motor

263 learning deficits (Donchin et al., 2012; Rabe et al., 2009). Cerebellar degeneration was less

264 pronounced in the inferior parts of the posterior lobe compared to earlier work (Hulst et al.,

265 2015), which could be explained by younger cerebellar participants in the current study, with

266 less severe ataxia scores. 


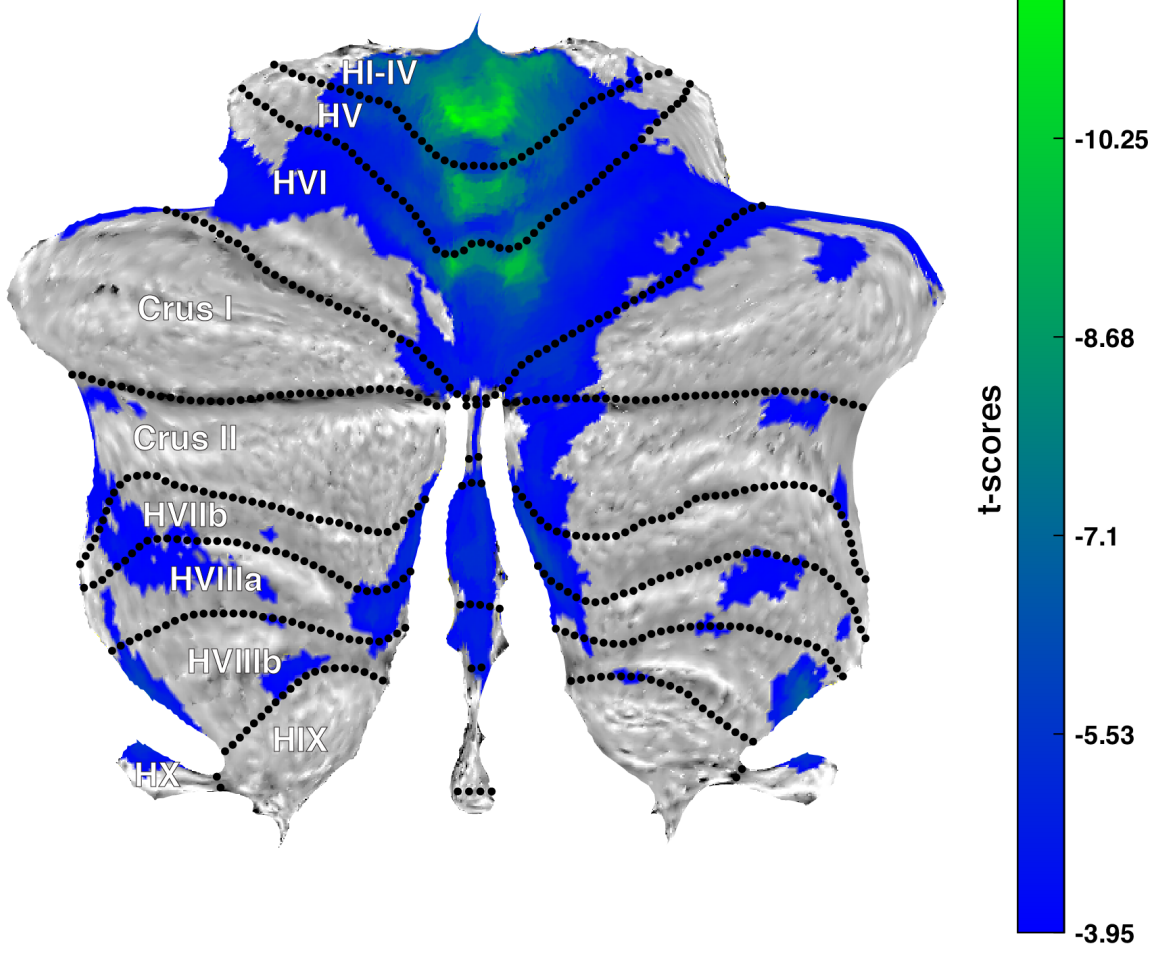

268 Figure 3: Flatmap of the cerebellum. Colors indicate the gray matter volume difference per voxel between 269 healthy participants and cerebellar participants in t-scores. Voxels that do not exceed the threshold (-3.95) are 270 not colored, low significant $t$-scores are colored blue, and high significant $t$-scores are colored green. Flatmap 271 template from Diedrichsen and Zotow, 2015.

\section{$272 \quad 3.2$ Average reaching directions}

273 The average reaching directions for each paradigm in control participants and cerebellar

274 participants are plotted in Figure 4A and 4C. Differences in mean reaching directions per

275 phase, paradigm and subject group were tested using the mixed-design model from the

276 methods section and the most important results are described below. 

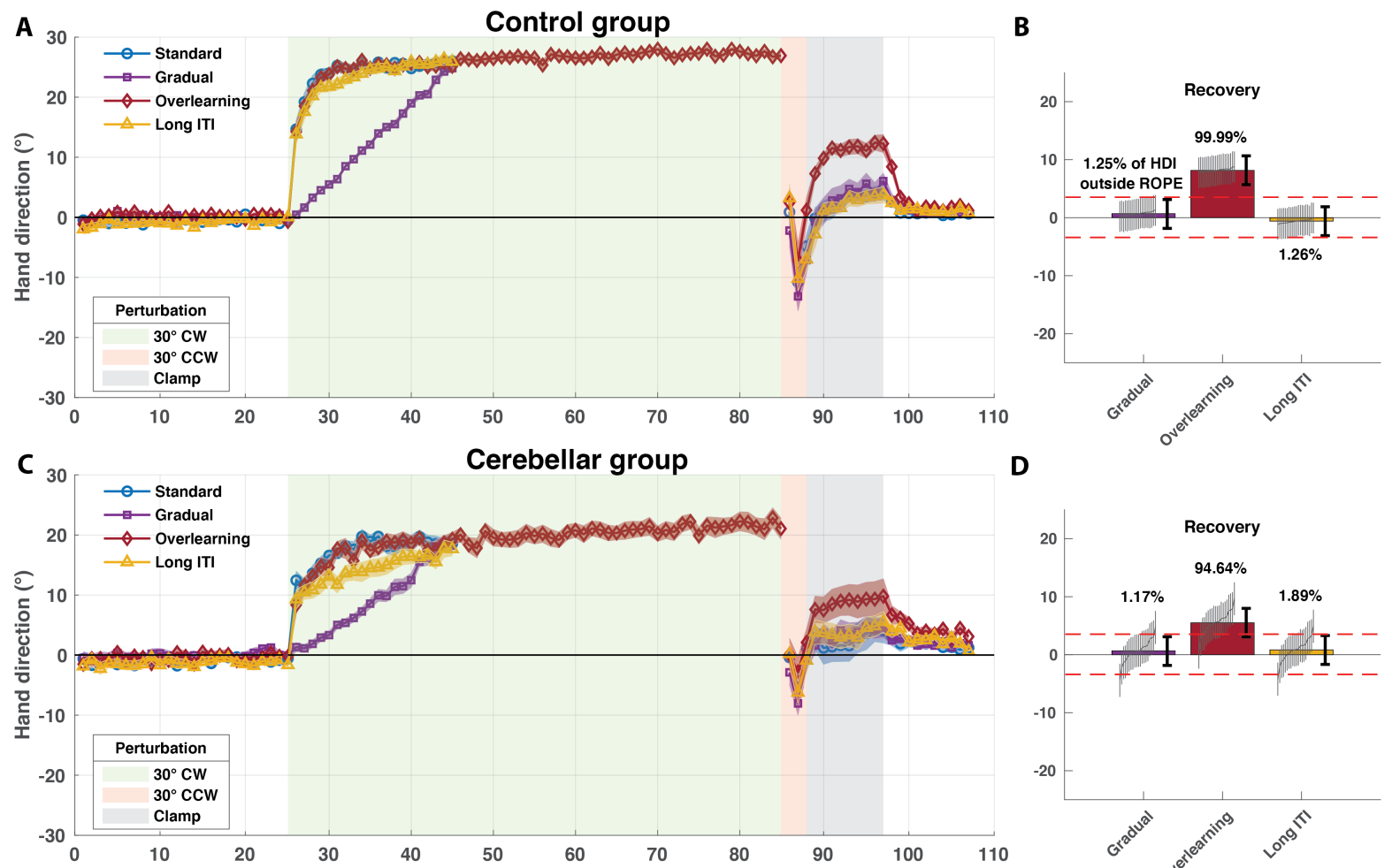

D

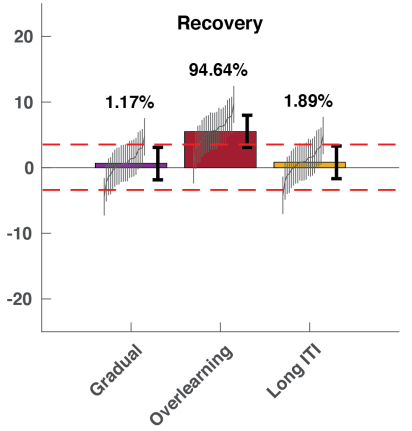

$\mathbf{E}$
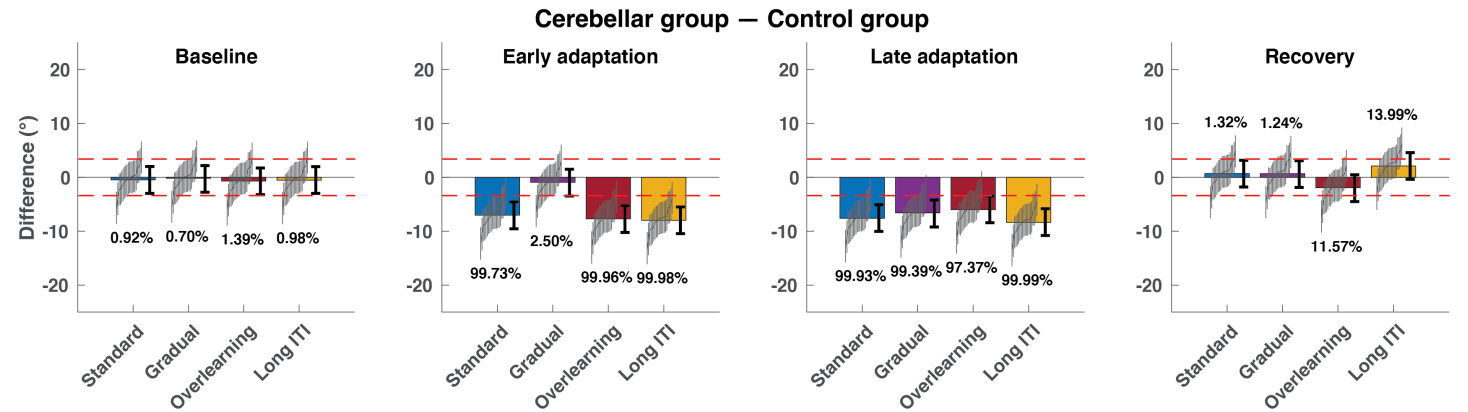

Figure 4: Average reaching directions of control participants and cerebellar participants. A) Reaching directions of control participants. Trials were binned per 6 trials. Shaded errorbars are mean \pm SEM. B) Difference in reaching directions between the standard paradigm and slow learning paradigms during the recovery phase of control participants. Thin errorbars indicate HDI of individual participants, thick errorbars indicate HDI of group. \% indicates percentage of HDI outside the ROPE. C) Reaching directions of cerebellar participants. D) Difference in reaching directions between the standard paradigm and slow learning paradigms during the recovery phase of cerebellar participants. E) Difference in reaching directions between cerebellar participants and control participants over all paradigms and phases of learning.

As expected, reaching directions of control participants and cerebellar participants are

practically straight during baseline in all training paradigms. When movements are perturbed

by a visuomotor rotation, control participants learn the perturbation quickly, almost

completely counteracting the rotation early and late in the adaptation set (barring the early

phase of the gradual paradigm). Cerebellar participants adapt more slowly and much less, 
counteracting about half of the rotation compared to healthy controls. In general, control participants counteract more of the perturbation than cerebellar participants, both early and

293 late in the adaptation set (Figure 4E).

294 Behaviorally, the most salient difference between paradigms in control participants exists

295 between the amount of spontaneous recovery in the standard paradigm versus the

296 overlearning paradigm, i.e., there is more spontaneous recovery after overlearning than

297 standard learning (Figure 4B). Similarly, the difference between the overlearning paradigm

298 and the standard paradigm in cerebellar participants is highly suggestive of more spontaneous

299 recovery after overlearning as well, though this difference is smaller and obscured by the

300 larger variability in the cerebellar patients (Figure 4D). We hypothesized that the additional

301 amount of spontaneous recovery in both groups could have two different causes. It might be

302 due to more slow learning resulting from the prolonged activation of the slow system.

303 Alternatively, the additional spontaneous recovery might occur despite similar levels of slow

304 learning through increased retention in the slow system. These hypotheses will be explored in

305 the next section.

$306 \quad 3.3$ Modelling

307 Since behavioral differences between the paradigms were mainly evident in overlearning and 308 standard learning, we focus on comparing these paradigms. The model results for the other 309 paradigms can be found as supplementary material. To assess how the differences in the 310 learning and retention rate between training paradigms affect motor output $\left(y_{t}\right)$ and the states $311\left(x_{t}^{F}\right.$ and $\left.x_{t}^{S}\right)$, posterior predictive plots were generated from multiple random draws $(\mathrm{n}=$ $31210,000)$ of the posterior distributions of each participant in the control and cerebellar group

313 (Figure 5). The model captures the structure of a subject's behavior: rapid learning is

314 followed by a plateau and the short period of counterperturbation leads to spontaneous 315 recovery. The model also captures the increased spontaneous recovery in the overlearning 
316 paradigm and the fact that this increase is much smaller for cerebellar patients than for

318 for both groups is shown by the blue bars in Figure $\mathbf{5 C}$ and 5F. The model predicts an

319 increase of $6.12^{\circ}$ in spontaneous recovery for controls and an increase of $2.04^{\circ}$ for cerebellar

320 patients.
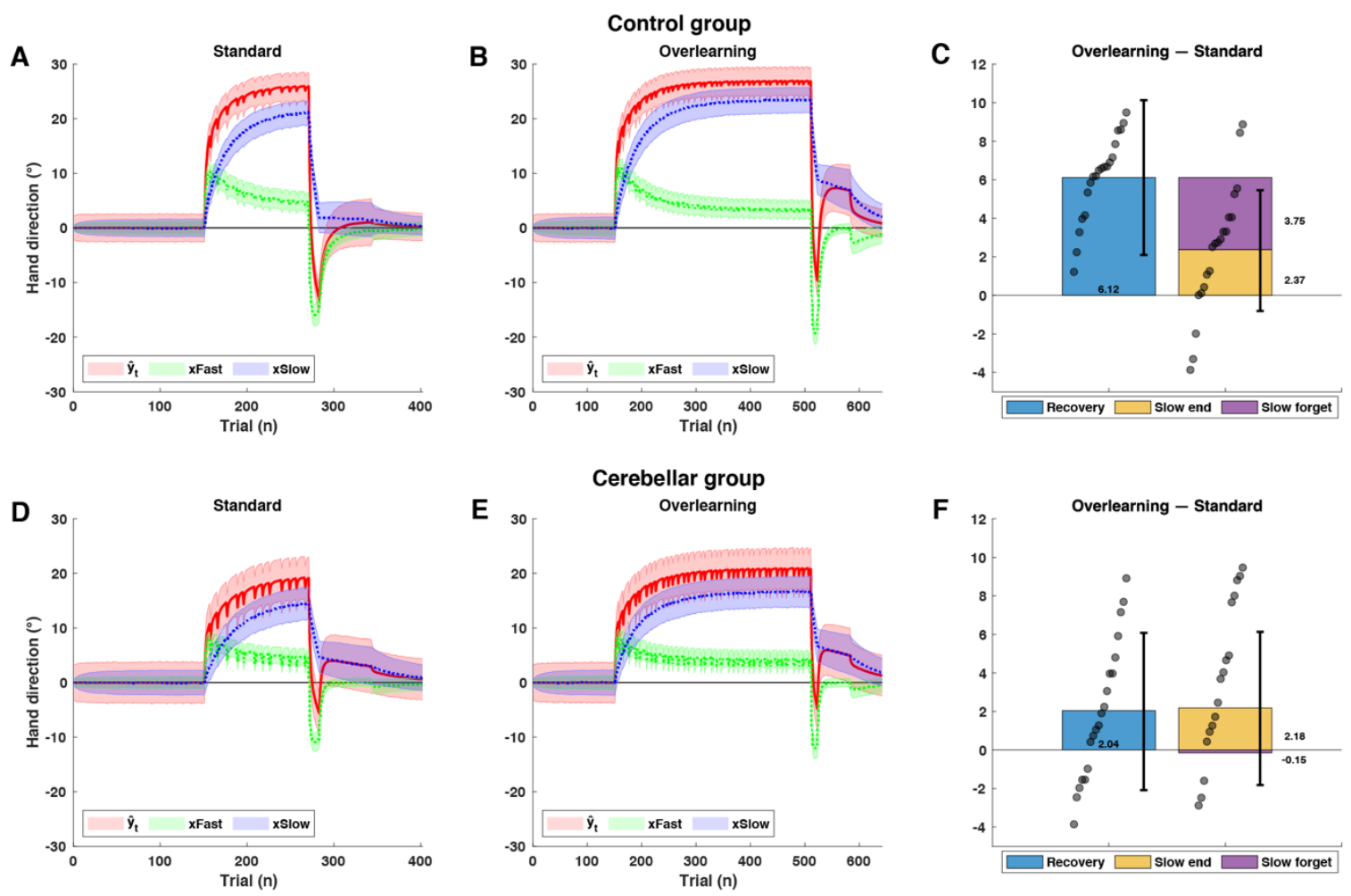

Figure 5: Posterior predictive plots of control participants in the A) standard and B) overlearning paradigm. The average model output $\left(\hat{y}_{t}\right)$ is displayed with a solid red line, the average fast state $\left(x_{t}^{F}\right)$ with a dotted green line, and the average slow state $\left(x_{t}^{S}\right)$ with a dotted blue line. The shaded errorbars indicate the variability around the average posterior predictive $\left(2.5^{\text {th }}\right.$ percentile $-97.5^{\text {th }}$ percentile of simulated data $)$. Panel C) shows the difference in spontaneous recovery of the slow system between the standard and overlearning paradigm (blue bar) and the difference in the amount of slow learning at the end of the adaptation set (yellow bar). The purple bar is the difference in the drop of slow learning after the counterperturbation trials between the standard and overlearning paradigm. Grey circles on the blue bar indicate individual differences in spontaneous recovery, the thick errorbar is the HDI of the group. Grey circles on the yellow bar indicate individual differences in slow learning at the end of adaptation, the thick errorbar is the HDI of the group. Panels D), E) and F) display the same plots for cerebellar participants instead.

333 The results of the model support the intuitive idea that spontaneous recovery reflects slow

334 learning. For both groups, fast learning is essentially 0 during the spontaneous recovery 
that increased spontaneous recovery reflects increased slow learning at the end of adaptation.

The yellow bar in Figure 5C shows the difference in the amount of slow learning in the model at the end of adaptation in the standard paradigm vs the overlearning paradigm. Slow

339 learning in controls is only $2.37^{\circ}$ higher in the overlearning paradigm than in the standard 340 paradigm, a difference similar to that in cerebellar patients $\left(2.18^{\circ}\right)$. Indeed, in controls, the 341 model predicts that increased spontaneous recovery reflects primarily a reduced drop in slow 342 learning after the counterperturbation trials (i.e., less forgetting of the slow system). As 343 depicted by the purple bar in Figure 5C, the slow forgetting for controls in the overlearning 344 paradigm is $3.75^{\circ}$ smaller than in the standard paradigm. Thus, for controls, about $61.3 \%$ of 345 the increased spontaneous recovery comes from a reduced drop in slow learning during the 346 counterperturbation phase and only $38.7 \%$ from increased buildup of slow learning during the 347 elongated adaptation set. In contrast, for cerebellar patients, practically all increased 348 spontaneous recovery is a result of added buildup of slow learning.

349 To understand how the model generated less slow forgetting after counterperturbation trials in 350 the overlearning paradigm in control participants, we examined how learning and retention 351 parameters changed between the standard and overlearning paradigm (Figure 6). As the 352 figure shows, the model suggests that, in controls, slow retention (ASlow) is higher in 353 overlearning than in the standard paradigm and the slow learning rate (BSlow) is actually less 354 (Figure 6A). This result is consistent across control participants and contrasts with the 355 learning rate and retention in the fast state, which are largely unchanged between the 356 paradigms. While the model also ascribes a decreased slow learning rate to some cerebellar 357 subjects, there are a few cerebellar subjects who do not show such a shift (Figure 6B). 

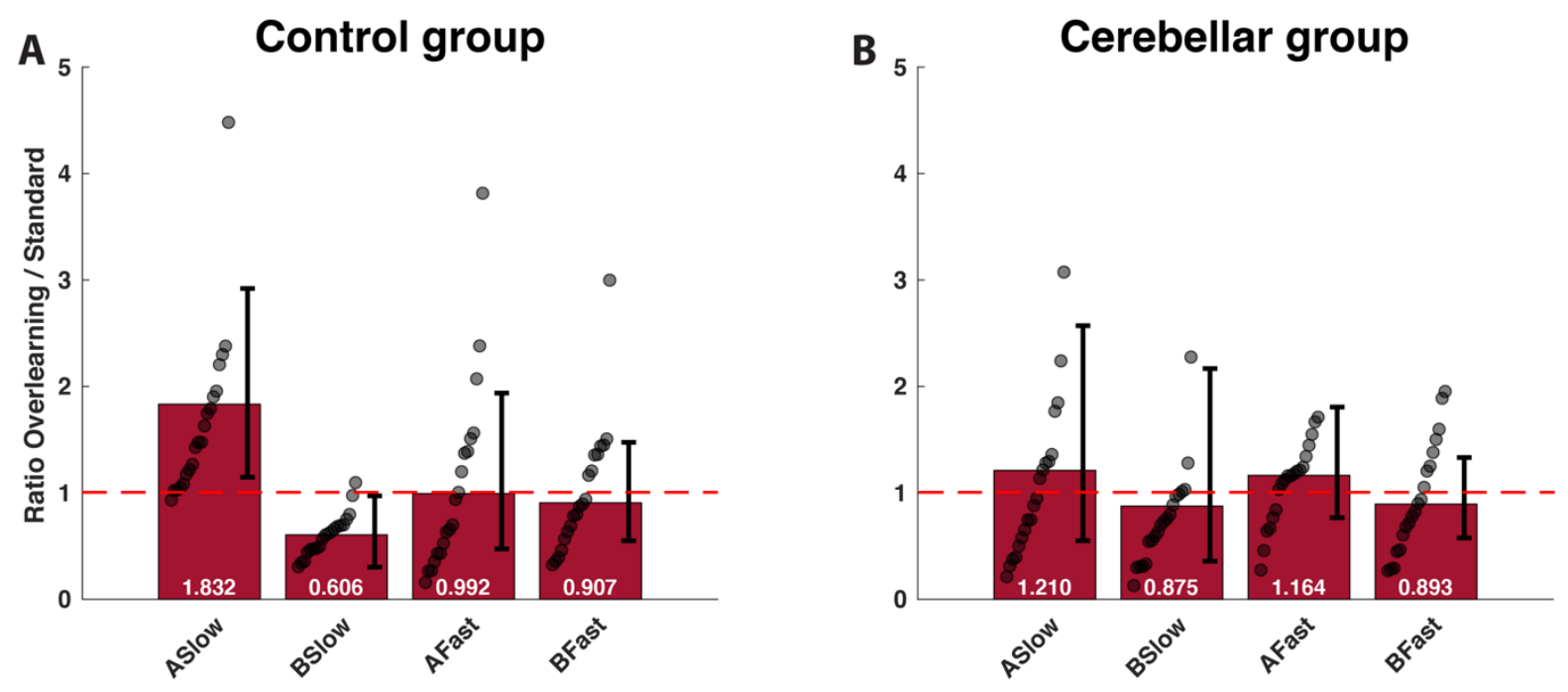

Figure 6: Change in learning and retention parameters between paradigms. The change in parameters is expressed as a ratio between the standard and overlearning paradigm. For A parameters the ratio was taken as $\frac{1-A_{\text {standard }}}{1-A_{\text {overlearning }}}$, for $B$ parameters the ratio was taken as $\frac{B_{\text {overlearning }}}{B_{\text {standard }}}$. This choice was made so that in both cases we are showing ratios of rates (forgetting rate and learning rate) and also so increased slow learning in overlearning would lead to larger numbers for both parameters. The gray circles represent ratios for individual participants, the thick black errorbar is the HDI of the population parameter. The red line indicates a ratio of 1 . A) Control participants. B) Cerebellar participants.

366 These changes in model parameters may be the cause of the change in model behavior

367 between the two tasks, but it is also possible that changes in model behavior reflect the 368 extended adaptation phase in the overlearning paradigm. To test the effect of the changes in 369 the model parameters on model behavior, we generated posterior predictive data for the overlearning paradigm using parameters from the standard paradigm and for the standard paradigm using parameters from the overlearning paradigm. The results of this are shown in

372 Figure 7. The figure shows that control participants have much smaller spontaneous recovery

373 in the overlearning paradigm when using parameters from the standard paradigm (Figure

374 7A). Similarly, spontaneous recovery in the standard paradigm is larger using parameters

375 from the overlearning paradigm (Figure 7B). Out of the total difference in the spontaneous

376 recovery of controls between paradigms, practically all of it seems to come from the

377 difference in parameters (Figure 7C). In other words, the changes in parameters primarily

378 cause reduced slow forgetting during the counterperturbation and they have little effect on 
379 level of the slow at the end of adaptation. A similar analysis of the model fit to cerebellar

380 patients produces different results (Figure 7D-F). In the case of cerebellar patients, there is

381 almost no difference in the amount of spontaneous recovery caused by swapping parameters.

382 Thus, the model does not provide a good explanation for the increase in spontaneous recovery

383 in the cerebellar patients.
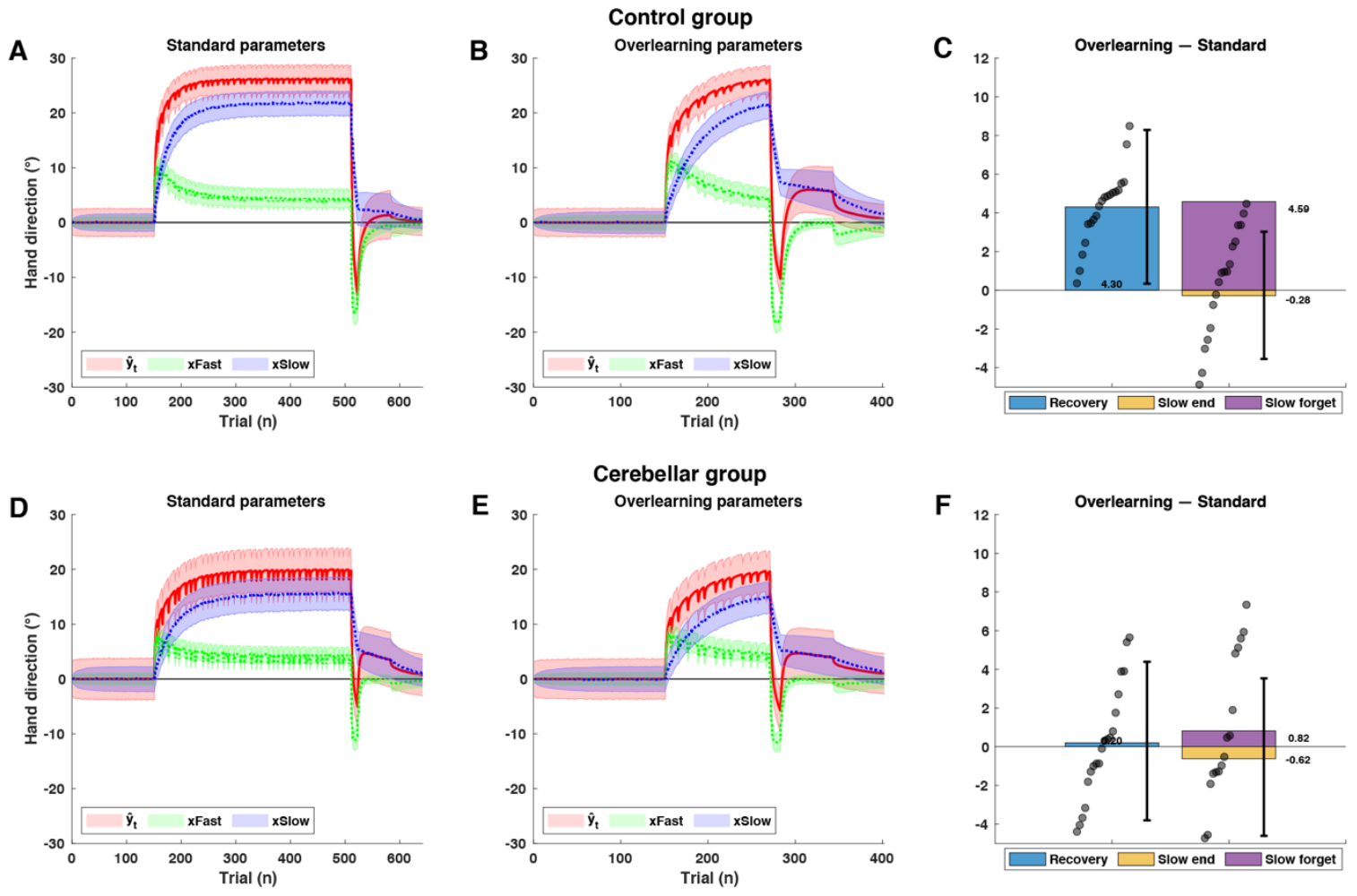

Figure 7: Posterior predictive plots generated using incongruent parameters. That is, the posterior predictive plots for the overlearning paradigm were generated from the parameters fit to the standard paradigm and vice versa. A) Control participants with standard parameters in the structure of the overlearning paradigm and B) overlearning parameters in the structure of the standard paradigm. The average model output $\left(\hat{y}_{t}\right)$ is displayed with a solid red line, the average fast state $\left(x_{t}^{F}\right)$ with a dotted green line, and the average slow state $\left(x_{t}^{S}\right)$ with a dotted blue line. The shaded errorbars indicate the variability around the average posterior predictive $\left(2.5^{\text {th }}\right.$ percentile $-97.5^{\text {th }}$ percentile of simulated data). Panel C) shows the difference in spontaneous recovery of the slow system between the standard parameters and overlearning parameters (blue bar) and the difference in the amount of slow learning at the end of the adaptation set (yellow bar). The purple bar is the difference in the drop of slow learning after the counterperturbation trials between the standard and overlearning parameters. Grey circles on the blue bar indicate individual differences in spontaneous recovery, the thick errorbar is the HDI of the group. Grey circles on the yellow bar indicate individual differences in slow learning at the end of adaptation, the thick errorbar is the HDI of the group. Panels D), E) and F) display the same plots for cerebellar 


\section{Discussion}

400 We tested 3 paradigms that have been thought to increase slow learning in both controls and cerebellar patients. For each we examined spontaneous recovery after a short counterperturbation, a phenomenon that has been taken as a hallmark of slow learning (Smith et al., 2006). We find that only one paradigm, overlearning, increased spontaneous recovery and that it did so in both controls and cerebellar patients (Figure 4). Intuitively, increased spontaneous recovery might been taken as a sign that the level of slow learning is higher at the end of adaptation. We tested this by fitting a two-state model of motor adaptation to our data using a Bayesian fitting procedure and generating posterior predictive data to model the

408 behavior of the subjects. Our model showed increased spontaneous recovery, especially in 409 control subjects (Figure 5). However, this did not result from a higher level of slow learning at the end of adaptation. Rather, overlearning led to a smaller drop in the slow learning during

411 the counterperturbation. This seemed to be because, in controls, the model parameter for

412 retention of slow learning (ASlow) was higher during overlearning than during the standard 413 paradigm and the model parameter for slow learning rate (BSlow) was lower during the 414 overlearning than in the standard paradigm (Figure 6). We verified that the change in 415 parameters was the primary driver of the change in model behavior by using parameters from 416 the standard model in the overlearning paradigm and vice-versa. This showed that increased 417 spontaneous recovery in the model came primarily from changes in the parameters rather than 418 the difference in the number of trials performed by the model (Figure 7). Our model was not 419 able to explain the increased spontaneous recovery in cerebellar patients and did not show 420 strong changes in the parameter values in cerebellar patients going from the standard 421 paradigm to overlearning. Taken together our results indicate that extended training - as used 422 in our overlearning paradigm - can increase the resilience of slow learning to a 
424 smaller in cerebellar patients than in controls. Modelling results suggest that the mechanism

425 of increased resilience may be different in cerebellar patients and controls.

426 Our primary result is that the overlearning task was the only paradigm that led to more

427 spontaneous recovery than the standard tasks. There was logic to thinking the other tasks

428 would also lead to greater spontaneous recovery. Gradual adaptation has long been thought to

429 decrease the rate at which retention washes away compared to abrupt adaptation

430 (Criscimagna-Hemminger et al., 2010; Huang and Shadmehr, 2009; Kagerer et al., 1997;

431 Kluzik et al., 2008; Michel et al., 2007; Wong and Shelhamer, 2011) although there have been

432 some studies that did not find such an effect (Klassen et al., 2005; Werner et al., 2014). A

433 recent paper suggests that the apparent effects on retention in the gradual condition actually

434 reflects secondary effects either of training duration or level of learning (Alhussein et al.,

435 2019). This is entirely consistent with our results. Our expectation regarding an effect of long

436 ITIs is rooted in the history showing increased retention following spaced learning compared

437 to massed learning (for a review see Smolen et al., 2016), that has been documented long ago

438 also in motor adaptation (Taub and Goldberg, 1973). Indeed, recent work has suggested that

439 long ITIs should drive stronger retention in adaptation (Kim et al., 2015; Zhou et al., 2017).

440 However, in these studies, effects are small and individual subject variability is large with

441 delays on the order of $15 \mathrm{sec}$. In both studies, details of the experimental protocol were also

442 quite different from our own. Thus, it might be possible to see effects with a long ITI, but it

443 would require delays that are quite long and a different task. In sum, our results are consistent

444 with other results in the literature raising doubts about whether either gradual adaptation or

445 long ITIs drive the slow learning system.

446 Overlearning - the continued practice once performance has plateaued - has long been known

447 to increase retention (for review see Driskell et al., 1992). The effect of the number of training

448 trials on retention of motor adaptation has been confirmed more recently (Joiner and Smith, 
2008; Yamada et al., 2019). Indeed, as mentioned, Alhussein (2019) showed that previously reported effects of gradual adaptation on slow learning are, to a large extent, mediated by the number of training trials. Our findings are consistent with these previous findings. However,

452 the effects shown previously are different from ours. First, Joiner and Smith (2008) and

453 Yamada (2019) both show increased retention after 24 hours, while Alhussein (2019)

454 measures the rate of decay of performance in a no feedback condition immediately after

455 training. Ours is the first paper to our knowledge to show an effect of overlearning on 456 spontaneous recovery.

457 Our second basic result is that spontaneous recovery was also increased in patients, although 458 in a manner that was less pronounced than in controls. Consistent with many previous

459 findings, cerebellar subjects learned slower than controls and reached a lower level of 460 adaptation at the end of the adaptation (Figure 4). During spontaneous recovery, their 461 performance was close to that of controls for all paradigms, including the overlearning. This 462 suggests that overlearning does cause some increased slow learning in patients as well as 463 controls.

464 Our third basic result comes from our modeling which suggests that overlearning changes the 465 parameters of the slow system in controls. Joiner and Smith (2008) ascribe most of the effect 466 of overlearning to an increase in the level of slow adaptation with increased number of

467 training trials. This contrasts with our interpretation: most of the effect reflects slow 468 adaptation becoming more resistant to change as overlearning progresses. The differences 469 may arise from the length of training in the two studies. Joiner and Smith (2008) show an 470 effect that begins to level off near 100 training trials and the group with the most training only 471 does 160 training trials. Our study had one group with around 108 training trials and another 472 with 324 training trials. Thus, it is possible that what they see reflects a slow rise in the level 
473 of the slow adaptation system, but that after this system reaches a plateau increased training

474 begins to change its responsiveness.

475 Model results also suggest that at the end of the standard paradigm, the slow system of

476 cerebellar patients has not yet reached plateau and some increase is still possible in the slow

477 learning system. The model suggests that cerebellar patients have less slow learning than

478 controls and more slow forgetting. Fast learning of cerebellar patients and controls is quite

479 similar, consistent with earlier experimental findings (Taylor et al., 2010; Wong et al., 2019).

480 The idea that the history of adaptation influences its dynamics is not new (Shadmehr and

481 Brashers-Krug, 1997). Our suggestion that overlearning may lead to increased resiliency in

482 the slow system is in line with previous research specifically suggesting that environmental

483 consistency affects adaptation dynamics (Avraham et al., 2019). Indeed, previous work has

484 also used changing fit of the two state model as evidence of changes in learning parameters

485 (Mawase et al., 2014).

486 Conclusions drawn from our research must be tentative due to a number of limitations. One

487 important caveat is that our design is a within subjects design with each subject doing all tasks

488 on the same day. We addressed this limitation in different ways. First, we used washout at the

489 end of each session to reduce transfer (Caithness et al., 2004; Krakauer et al., 2005; Nguyen et

490 al., 2019; but see also Kitago et al., 2013; Villalta et al., 2015). Second, order of the tasks and

491 perturbation direction was counterbalanced between subjects and we tested for order effects

492 (see supplementary materials). We do not believe that our central result is sensitive to possible

493 inter-session effects. A second concern is that subtle details of the task - such as the fact that

494 the gradual adaptation group did not spend any time in the plateau or that the long ITIs may

495 have been too short -- may have specifically influenced our results. This possibility cannot be

496 ruled out. A third concern is one that is characteristic of all patient studies: patients are

497 variable, disease etiology is complex, and we are specifically studying chronic lesions. 
498 Perhaps the most salient limitation of our study is in the interpretation of the modeling. The

499 modeling work cannot be conclusive, and alternative explanations exist. Overlearning may

500 engage additional learning mechanisms that are not accounted for by the two-state model. For

501 instance, Therrien et al., (2016) has shown that reinforcement learning can be selectively

502 engaged in the adaptation task, and that this can affect the way subjects behave during error

503 clamps (Shmuelof et al., 2012). Additional mechanisms to consider are use-dependent

504 learning (Diedrichsen et al., 2010) or model-free learning (Huang et al., 2011). Finally, a

505 recently published model uses dynamic formation of specific adaptation memory to explain

506 many of the dynamics of adaptation (Heald et al., 2020). Thus, our findings and modeling

507 work lend credence to the idea that extra practice makes learning more resilient, but they do

508 not conclusively identify the mechanisms based on comparison of alternative models.

509 In sum, our research shows that spontaneous recovery is specifically affected by overtraining

510 in cerebellar patients and controls. We hypothesize that, in controls, this is primarily driven by

511 changes in the dynamics of slow adaptation while in cerebellar patients it reflects changes in

512 the amount of slow adaptation achieved. That is, while residual slow learning does exist in

513 cerebellar patients, we do not see evidence that the primary cerebellar deficit can be improved

514 by overtraining paradigms. 


\section{References}

516 Alhussein, L., Hosseini, E.A., Nguyen, K.P., Smith, M.A., and Joiner, W.M. (2019). Dissociating effects of error size, training duration, and amount of adaptation on the ability to retain motor memories. J. Neurophysiol. 122, 2027-2042.

Avraham, G., Keizman, M., and Shmuelof, L. (2019). Environmental consistency modulation of error sensitivity during motor adaptation is explicitly controlled. J. Neurophysiol. 123, 5769.

Caithness, G., Osu, R., Bays, P., Chase, H., Klassen, J., Kawato, M., Wolpert, D.M., and Flanagan, J.R. (2004). Failure to Consolidate the Consolidation Theory of Learning for Sensorimotor Adaptation Tasks. J. Neurosci. 24, 8662-8671.

Coltman, S.K., Cashaback, J.G.A., and Gribble, P.L. (2019). Both fast and slow learning processes contribute to savings following sensorimotor adaptation. J. Neurophysiol. 121, $1575-1583$.

Criscimagna-Hemminger, S.E., Bastian, A.J., and Shadmehr, R. (2010). Size of error affects cerebellar contributions to motor learning. J. Neurophysiol. 103, 2275-2284.

530 Diedrichsen, J., and Zotow, E. (2015). Surface-Based Display of Volume-Averaged Cerebellar Imaging Data. PLOS ONE 10, e0133402.

532 Diedrichsen, J., Balsters, J.H., Flavell, J., Cussans, E., and Ramnani, N. (2009). A

533 probabilistic MR atlas of the human cerebellum. NeuroImage 46, 39-46.

534 Diedrichsen, J., White, O., Newman, D., and Lally, N. (2010). Use-Dependent and Error-

535 Based Learning of Motor Behaviors. J. Neurosci. 30, 5159-5166.

536 Donchin, O., Rabe, K., Diedrichsen, J., Lally, N., Schoch, B., Gizewski, E.R., and Timmann, D. (2012). Cerebellar Regions Involved in Adaptation to Force Field and Visuomotor

538 Perturbation. J. Neurophysiol. 107, 134-147.

539 Driskell, J.E., Willis, R.P., and Copper, C. (1992). Effect of overlearning on retention. J.

540 Appl. Psychol. 77, 615-622.

541 Fonteyn, E.M.R., Keus, S.H.J., Verstappen, C.C.P., Schöls, L., de Groot, I.J.M., and van de

542 Warrenburg, B.P.C. (2014). The effectiveness of allied health care in patients with ataxia: a

543 systematic review. J. Neurol. 261, 251-258.

544 Forano, M., and Franklin, D.W. (2020). Timescales of motor memory formation in dual545 adaptation. BioRxiv 698167.

546 Gibo, T.L., Criscimagna-Hemminger, S.E., Okamura, A.M., and Bastian, A.J. (2013). 547 Cerebellar motor learning: are environment dynamics more important than error size? J. 548 Neurophysiol. 110, 322-333.

549 Gomez, C.M., Thompson, R.M., Gammack, J.T., Perlman, S.L., Dobyns, W.B., Truwit, C.L., 550 Zee, D.S., Clark, H.B., and Anderson, J.H. (1997). Spinocerebellar ataxia type 6: Gaze-

551 evoked and vertical nystagmus, Purkinje cell degeneration, and variable age of onset. Ann.

552 Neurol. 42, 933-950. 
553 Hatakenaka, M., Miyai, I., Mihara, M., Yagura, H., and Hattori, N. (2012). Impaired Motor

554 Learning by a Pursuit Rotor Test Reduces Functional Outcomes During Rehabilitation of

555 Poststroke Ataxia. Neurorehabil. Neural Repair 26, 293-300.

556 Heald, J.B., Lengyel, M., and Wolpert, D.M. (2020). Contextual inference underlies the

557 learning of sensorimotor repertoires. BioRxiv 2020.11.23.394320.

558 Huang, V.S., and Shadmehr, R. (2009). Persistence of Motor Memories Reflects Statistics of

559 the Learning Event. J. Neurophysiol. 102, 931-940.

560 Huang, V.S., Haith, A., Mazzoni, P., and Krakauer, J.W. (2011). Rethinking Motor Learning

561 and Savings in Adaptation Paradigms: Model-Free Memory for Successful Actions Combines

562 with Internal Models. Neuron 70, 787-801.

563 Hull, C. (2020). Prediction signals in the cerebellum: beyond supervised motor learning.

564 ELife 9, e54073.

565 Hulst, T., van der Geest, J.N., Thürling, M., Goericke, S., Frens, M. a., Timmann, D., and

566 Donchin, O. (2015). Ageing shows a pattern of cerebellar degeneration analogous, but not

567 equal, to that in patients suffering from cerebellar degenerative disease. NeuroImage 116,

568 196-206.

569 Ilg, W., Bastian, A.J., Boesch, S., Burciu, R.G., Celnik, P., Claa??en, J., Feil, K., Kalla, R.,

570 Miyai, I., Nachbauer, W., et al. (2014). Consensus paper: Management of degenerative

571 cerebellar disorders. Cerebellum 13, 248-268.

572 Jayadev, S., and Bird, T.D. (2013). Hereditary ataxias: Overview. Genet. Med. 15, 673-683.

573 Joiner, W.M., and Smith, M.A. (2008). Long-Term Retention Explained by a Model of Short-

574 Term Learning in the Adaptive Control of Reaching. J. Neurophysiol. 100, 2948-2955.

575 Kagerer, F.A., Contreras-Vidal, J.L., and Stelmach, G.E. (1997). Adaptation to gradual as

576 compared with sudden visuo-motor distortions. Exp. Brain Res. 115, 557-561.

577 Kim, S., Oh, Y., and Schweighofer, N. (2015). Between-Trial Forgetting Due to Interference and Time in Motor Adaptation. PLOS ONE 10, e0142963.

579 Kitago, T., Ryan, S.L., Mazzoni, P., Krakauer, J.W., and Haith, A.M. (2013). Unlearning

580 versus savings in visuomotor adaptation: comparing effects of washout, passage of time, and

581 removal of errors on motor memory. Front. Hum. Neurosci. 7.

582 Klassen, J., Tong, C., and Flanagan, J.R. (2005). Learning and recall of incremental kinematic and dynamic sensorimotor transformations. Exp. Brain Res. 164, 250-259.

584 Kluzik, J., Diedrichsen, J., Shadmehr, R., and Bastian, A.J. (2008). Reach Adaptation: What

585 Determines Whether We Learn an Internal Model of the Tool or Adapt the Model of Our

586 Arm? J. Neurophysiol. 100, 1455-1464.

587 Krakauer, J.W., Ghez, C., and Ghilardi, M.F. (2005). Adaptation to visuomotor

588 transformations: consolidation, interference, and forgetting. J. Neurosci. Off. J. Soc. Neurosci.

589 25, 473-478. 
590 Krakauer, J.W., Hadjiosif, A.M., Xu, J., Wong, A.L., and Haith, A.M. (2019). Motor

591 Learning. Compr. Physiol. 9, 613-663.

592 Kruschke, J. (2010). Doing Bayesian Data Analysis.

593 Mariotti, C., Fancellu, R., and Donato, S. (2005). An overview of the patient with ataxia. J.

594 Neurol. 252, 511-518.

595 Maschke, M., Gomez, C.M., Ebner, T.J., and Konczak, J. (2004). Hereditary cerebellar ataxia 596 progressively impairs force adaptation during goal-directed arm movements. J. Neurophysiol.

597 91, 230-238.

598 Matilla-Dueñas, A., Ashizawa, T., Brice, A., Magri, S., McFarland, K.N., Pandolfo, M., Pulst, 599 S.M., Riess, O., Rubinsztein, D.C., Schmidt, J., et al. (2014). Consensus Paper: Pathological 600 Mechanisms Underlying Neurodegeneration in Spinocerebellar Ataxias. The Cerebellum 13, 601 269-302.

602 Mawase, F., Shmuelof, L., Bar-Haim, S., and Karniel, A. (2014). Savings in locomotor 603 adaptation explained by changes in learning parameters following initial adaptation. J. 604 Neurophysiol. 111, 1444-1454.

605 McDougle, S.D., Bond, K.M., and Taylor, J. a. (2015). Explicit and Implicit Processes 606 Constitute the Fast and Slow Processes of Sensorimotor Learning. J. Neurosci. 35, 95686079579.

608 Michel, C., Pisella, L., Prablanc, C., Rode, G., and Rossetti, Y. (2007). Enhancing 609 Visuomotor Adaptation by Reducing Error Signals: Single-step (Aware) versus Multiple-step 610 (Unaware) Exposure to Wedge Prisms. J. Cogn. Neurosci. 19, 341-350.

611 Nguyen, K.P., Zhou, W., McKenna, E., Colucci-Chang, K., Bray, L.C.J., Hosseini, E.A., 612 Alhussein, L., Rezazad, M., and Joiner, W.M. (2019). The 24-h savings of adaptation to novel 613 movement dynamics initially reflects the recall of previous performance. J. Neurophysiol. $614 \quad 122,933-946$.

615 Oldfield, R.C. (1971). The assessment and analysis of handedness: the Edinburgh inventory. 616 Neuropsychologia 9, 97-113.

617 Petitet, P., O'Reilly, J.X., and O'Shea, J. (2018). Towards a neuro-computational account of 618 prism adaptation. Neuropsychologia 115, 188-203.

619 Plummer, M. (2003). JAGS: A program for analysis of Bayesian graphical models using 620 Gibbs sampling. Proc. 3rd Int. Workshop Distrib. Stat. Comput. DSC 2003 20-22.

621 Rabe, K., Livne, O., Gizewski, E.R., Aurich, V., Beck, a, Timmann, D., and Donchin, O. 622 (2009). Adaptation to visuomotor rotation and force field perturbation is correlated to 623 different brain areas in patients with cerebellar degeneration. J. Neurophysiol. 101, 19616241971.

625 Schlerf, J.E., Xu, J., Klemfuss, N.M., Griffiths, T.L., and Ivry, R.B. (2013). Individuals with 626 cerebellar degeneration show similar adaptation deficits with large and small visuomotor 627 errors. J. Neurophysiol. 109, 1164-1173. 
628 Schmahmann, J.D., and Sherman, J.C. (1998). The cerebellar cognitive affective syndrome.

629 Brain 121, 561-579.

630 Schmitz-Hübsch, T., Du Montcel, S.T., Baliko, L., Berciano, J., Boesch, S., Depondt, C.,

631 Giunti, P., Globas, C., Infante, J., Kang, J.S., et al. (2006). Scale for the assessment and rating

632 of ataxia. Neurology 66, 1717-1720.

633 Scoles, D.R., Meera, P., Schneider, M.D., Paul, S., Dansithong, W., Figueroa, K.P., Hung, G.,

634 Rigo, F., Bennett, C.F., Otis, T.S., et al. (2017). Antisense oligonucleotide therapy for

635 spinocerebellar ataxia type 2. Nature 544, 362-366.

636 Shadmehr, R., and Brashers-Krug, T. (1997). Functional Stages in the Formation of Human

637 Long-Term Motor Memory. J. Neurosci. 17, 409-419.

638 Shmuelof, L., Huang, V.S., Haith, A.M., Delnicki, R.J., Mazzoni, P., and Krakauer, J.W.

639 (2012). Overcoming motor "forgetting" through reinforcement of learned actions. J. Neurosci.

640 Off. J. Soc. Neurosci. 32, 14617-14621.

641 Sing, G., Najafi, B., Adewuyi, A., and Smith, M. (2009). A mechanism for the spacing effect:

642 Competitive inhibition between adaptive processes explains the increase in motor skill

643 retention associated with prolonged inter-trial spacing. Adv. Comput. Mot. Control Chic. IL.

644 Smith, M.A., Ghazizadeh, A., and Shadmehr, R. (2006). Interacting Adaptive Processes with

645 Different Timescales Underlie Short-Term Motor Learning. PLoS Biol 4, e179.

646 Smolen, P., Zhang, Y., and Byrne, J.H. (2016). The right time to learn: mechanisms and

647 optimization of spaced learning. Nat. Rev. Neurosci. 17, 77-88.

648 Taig, E., Küper, M., Theysohn, N., Timmann, D., and Donchin, O. (2012). Deficient use of

649 visual information in estimating hand position in cerebellar patients. J. Neurosci. Off. J. Soc.

650 Neurosci. 32, 16274-16284.

651 Taub, E., and Goldberg, I.A. (1973). Prism Adaptation: Control of Intermanual Transfer by

652 Distribution of Practice. Science 180, 755-757.

653 Taylor, J.A., Klemfuss, N.M., and Ivry, R.B. (2010). An Explicit Strategy Prevails When the

654 Cerebellum Fails to Compute Movement Errors. The Cerebellum 9, 580-586.

655 Therrien, A.S., Wolpert, D.M., and Bastian, A.J. (2016). Effective reinforcement learning 656 following cerebellar damage requires a balance between exploration and motor noise. Brain $657 \quad 139,101-114$.

658 Timmann, D., Konczak, J., Ilg, W., Donchin, O., Hermsdörfer, J., Gizewski, E.R., and 659 Schoch, B. (2009). Current advances in lesion-symptom mapping of the human cerebellum. 660 Neuroscience 162, 836-851.

661 Trouillas, P., Takayanagi, T., Hallett, M., Currier, R.D., Subramony, S.H., Wessel, K., Bryer, 662 A., Diener, H.C., Massaquoi, S., Gomez, C.M., et al. (1997). International Cooperative Ataxia 663 Rating Scale for pharmacological assessment of the cerebellar syndrome. J. Neurol. Sci. 145, $664205-211$. 
665 Tseng, Y.-W., Diedrichsen, J., Krakauer, J.W., Shadmehr, R., and Bastian, A.J. (2007).

666 Sensory Prediction Errors Drive Cerebellum-Dependent Adaptation of Reaching. J.

667 Neurophysiol. 98, 54-62.

668 Villalta, J.I., Landi, S.M., Fló, A., and Della-Maggiore, V. (2015). Extinction interferes with

669 the retrieval of visuomotor memories through a mechanism involving the sensorimotor cortex.

670 Cereb. Cortex N. Y. N 1991 25, 1535-1543.

671 van der Vliet, R., Frens, M.A., de Vreede, L., Jonker, Z.D., Ribbers, G.M., Selles, R.W., van

672 der Geest, J.N., and Donchin, O. (2018). Individual Differences in Motor Noise and

673 Adaptation Rate Are Optimally Related. Eneuro 5, ENEURO.0170-18.2018.

674 Werner, S., Schorn, C.F., Bock, O., Theysohn, N., and Timmann, D. (2014). Neural correlates

675 of adaptation to gradual and to sudden visuomotor distortions in humans. Exp. Brain Res.

$676232,1145-1156$.

677 Williams, E. (1949). Experimental designs balanced for the estimation of residual effects of

678 treatments. Aust. J. Chem. 2, 149-168.

679 Wong, A.L., and Shelhamer, M. (2011). Saccade adaptation improves in response to a

680 gradually introduced stimulus perturbation. Neurosci. Lett. 500, 207-211.

681 Wong, A.L., Marvel, C.L., Taylor, J.A., and Krakauer, J.W. (2019). Can patients with

682 cerebellar disease switch learning mechanisms to reduce their adaptation deficits? Brain 142,

$683662-673$.

684 Yamada, C., Itaguchi, Y., and Fukuzawa, K. (2019). Effects of the amount of practice and

685 time interval between practice sessions on the retention of internal models. PLOS ONE 14,

686 e0215331.

687 Zarahn, E., Weston, G.D., Liang, J., Mazzoni, P., and Krakauer, J.W. (2008). Explaining

688 savings for visuomotor adaptation: linear time-invariant state-space models are not sufficient.

689 J. Neurophysiol. 100, 2537-2548.

690 Zhou, W., Fitzgerald, J., Colucci-Chang, K., Murthy, K.G., and Joiner, W.M. (2017). The

691 temporal stability of visuomotor adaptation generalization. J. Neurophysiol. 118, 2435-2447.

692 